\title{
Configuração espacial da Dengue no contexto socioeconômico de Alfenas/MG: Retrato de uma década
}

\section{Spatial configuration of the Dengue in the socioeconomic context of Alfenas-MG: Portrait of a decade}

\author{
Murilo C. do Nascimento ${ }^{1}$ Antonio L. Rodrigues-Júnior², Denis de O. Rodrigues³
}

\begin{abstract}
RESUMO
Introdução: A relação entre dengue e fatores socioeconômicos é objeto de controvérsia e não há registro de estudos em Alfenas-MG que tenham descrito tal interação na escala municipal. Objetivos: Caracterizar e agrupar os setores censitários de Alfenas-MG em níveis socioeconômicos e descrever a distribuição dos casos de dengue pelo território. Métodos: Estudo transversal em que foram utilizadas variáveis demográficas, estatística multivariada e análise de agrupamentos para gerar parâmetros de estratificação socioeconômica. Os casos confirmados de dengue clássico entre os anos de 2001 e 2010 foram geocodificados para estimar a densidade de pontos pela Técnica de Kernel e descrever a distribuição espacial da doença pelos clusters socioeconômicos. Resultados: Foram obtidos quatro clusters que agruparam as unidades censitárias em níveis socioeconômicos, sendo o número 1 considerado como melhor, o número 4 como pior, e os extratos 2 e 3 como níveis intermediários. Os locais de residência dos casos formaram diversos aglomerados, com distribuição aleatória pelo território, de forma indiferente aos níveis socioeconômicos. Ou seja, foi possível visualizar concentrações de casos de dengue tanto nas regiões com melhor perfil socioeconômico quanto nas áreas menos favorecidas quanto ao saneamento ambiental, condições de renda, escolaridade e adensamento populacional. Conclusão: Os resultados apontam que não houve relação alguma entre o padrão de distribuição geográfica da doença com os diferentes agrupamentos socioeconômicos do Município para o período estudado.
\end{abstract}

Palavras-chave: Dengue. Fatores Socioeconômicos. Análise Espacial. Estudos Transversais. Análise Multivariada. Governo Local.

\begin{abstract}
Introduction: The relation between dengue and socioeconomic factors is object of controversy and there's no record of studies in Alfenas-MG that had described such interaction in a municipal scale. Objectives: Characterize and group the census sectors of Alfenas-MG in socioeconomic levels and describe the distribution of the dengue cases around the area. Methods: Transversal study, in which were used demographic variables, multivariate statistics and analysis of the group to generate parameters of socioeco-
\end{abstract}

1. Docente da Universidade Federal de Alfenas, UNIFAL-MG. Doutorando do Programa de Pós-Graduação em Saúde na Comunidade da Faculdade de Medicina de Ribeirão Preto, FMRP, Universidade de São Paulo, USP.

2. Docente da Faculdade de Medicina de Ribeirão Preto, FMRP, Universidade de São Paulo, USP.

3. Servidor do Setor de Vigilância Epidemiológica da Prefeitura Municipal de Alfenas-MG.
Correspondência: Murilo César do Nascimento Rua Gabriel Monteiro da Silva, 700. Centro CEP: 37.130-000, Alfenas-MG murilo@unifal-mg.edu.br

Artigo recebido em 25/11/2013 Aprovado para publicação em 12/09/2014 
nomic stratification. Confirmed cases of classic dengue between the years of 2001 to 2010 were geocoded to estimate the density of points by the Kernel's Technique and describe the spatial distribution of the disease by the socioeconomic clusters. Results: There were obtained four clusters that grouped the census units in socioeconomic levels, in which the number 1 is regarded as the best, and the number 4 the worst, and the extracts 2 and 3 as intermediary levels. The residency's area from the cases made several groups, with a random distribution around the territory, in an indifferent way to the socioeconomic results. The concentration of points varied both located in sectors with better socioeconomic condition and in less privileged areas, as the middle of census areas with distinct levels. Conclusion: The results points toward the direction that there were no relations at all between the levels of geographic distribution of the disease with the different socioeconomic groups of the City to the studied period.

Key-words: Dengue. Socioeconomic Factors. Spatial Analysis. Cross-Sectional Studies. Multivariate Analysis. Local Government.

\section{Introdução}

No início do século XXI, quando os grandes problemas de saúde são essencialmente públicos, ${ }^{1}$ as desigualdades sociais têm se mantido no cenário mundial, com destaque acentuado nos países em desenvolvimento. ${ }^{2}$ No Brasil, tais disparidades configuramse relevantes, principalmente diante das evidências sobre o papel da situação socioeconômica na determinação da saúde. ${ }^{3,4}$ Por outro lado, independente da escala geográfica, o mecanismo exato de relação entre os fatores socioeconômicos e a produção de doenças nem sempre é tão claro, e os agravos à saúde são percebidos como "causa e o efeito, ao mesmo tempo, da pobreza, da desnutrição, das más condições de habitação e da ignorância". 5

No caso da reemergência e da disseminação da dengue não é diferente. Apesar das causas da mudança no comportamento epidemiológico da dengue ainda não estarem totalmente esclarecidas, é notável a associação de fatores demográficos, ecológicos e socioeconômicos à expansão da doença. ${ }^{6}$ Entretanto, a relação entre transmissão de dengue e níveis socioeconômicos é objeto de controvérsia, como apresentado em vários estudos com resultados discordantes. ${ }^{7,8}$

Em Alfenas-MG, não há registros de pesquisas cujo propósito tenha sido de investigar a configuração espacial da dengue e sua relação com os fatores sociais, ambientais e econômicos na escala municipal. Diante dessa carência e considerando a importância de se conhecer um pouco mais sobre este agravo no nível local é que este trabalho objetivou caracterizar e agrupar os setores censitários do Município em níveis socioeconômicos e descrever a distribuição dos casos de dengue pelo território, de 2001 a 2010. A divulgação dos resultados justifica-se por retratar aspectos epidemiológicos, geográficos e sociais de uma década, que podem contribuir para o enfrentamento local da doença.

\section{Métodos}

\subsection{Delineamento e área do estudo}

Tratou-se de um estudo transversal, com abordagem descritiva, que utilizou estatística multivariada e análise espacial em saúde. O Município de Alfenas localiza-se a $45^{\circ} 56^{\prime} 50^{\prime \prime}$ W de longitude e $21^{\circ} 25^{\prime} 45^{\prime}$ 'S de latitude, na região Sul do Estado de Minas Gerais, a cerca de $340 \mathrm{~km}$ da capital mineira Belo Horizonte - e 306 km de São Paulo-SP. Ocupa uma área de $849,2 \mathrm{Km}^{2}$ de extensão, com altitude máxima $888 \mathrm{~m}$ acima do nível do mar e a mínima de 768 m, na represa de Furnas. Articula-se em uma malha rodoviária com três estradas federais (BR-491, BR-267 e BR-369), algumas estaduais (MG-179, MG184, MG-453 e MG-879), e diversas municipais. Contém 75.214 habitantes, segundo estimativas populacionais para o TCU do Instituto Brasileiro de Geografia e Estatística - IBGE. ${ }^{9}$

\subsection{Coleta de dados}

Os dados sobre as notificações de casos autóctones de dengue em Alfenas-MG, referentes ao período de janeiro de 2001 a dezembro de 2010 foram obtidos no banco de dados descentralizado do Sistema de Informação de Agravos de Noticação - SINAN -, no Setor de Vigilância Epidemiológica da Secretaria de Saúde do Município. Para a definição dos casos de dengue este estudo considerou os registros de casos residentes e confirmados por exame laboratorial na detecção de anticorpos IgM para o antígeno da 
dengue no soro e os confirmados por vínculo clínicoepidemiológico com outro caso confirmado.

Já para obtenção de dados socioeconômicos foram calculados alguns parâmetros a partir de variáveis referentes ao saneamento ambiental, às condições de renda, à escolaridade e ao adensamento populacional, disponíveis no Censo Demográfico de $2000,{ }^{10}$ a saber:

- Média dos anos de instrução das pessoas responsáveis pelos domicílios;

- Média dos anos de instrução das mulheres responsáveis pelos domicílios;

- Renda média (em reais) das pessoas responsáveis pelos domicílios;

- Renda média (em reais) das mulheres responsáveis pelos domicílios;

- Proporção de pessoas responsáveis analfabetas (\%);

- Proporção de mulheres responsáveis analfabetas (\%);

- Proporção de domicílios com 5 ou mais moradores $(\%)$

- Proporção de domicílios com água encanada (\%);

- Proporção de domicílios com esgotamento sanitário $(\%)$

- Proporção de domicílios com coleta regular de lixo $(\%)$.

A utilização desses parâmetros se fundamentou em trabalhos que estudaram a relação entre a ocorrência de duas importantes doenças transmissíveis e o contexto socioeconômico numa escala submunicipal. ${ }^{71}$ Em contrapartida, a escolha dos setores censitários como objetos de estudo esteve fundamentada numa experiência que considerou esse nível de agregação por ser delimitado geograficamente e por apresentar características homogêneas. ${ }^{7}$

\subsection{Análise multivariada: análise de agrupa- mentos}

Uma vez definidos os parâmetros que caracterizariam os setores censitários do Município, optouse por trabalhar com Estatística Multivariada, para agrupar tais objetos geográficos em diferentes extratos socioeconômicos, por meio do método do Agrupamento Hierárquico de Ward. ${ }^{12}$ Análise multivariada pode ser entendida como "qualquer abordagem analítica que considere o comportamento de muitas variáveis simultaneamente" ${ }^{13}$ Nesse contexto, análi- se de agrupamentos é o termo utilizado para definir um grupo de técnicas multivariadas em que o objetivo principal é agregar objetos, levando em conta as suas características. ${ }^{14}$

O interesse em agrupar objetos, em determinadas situações, pode ser alterado para a intenção de agrupar variáveis. ${ }^{15}$ De qualquer modo, há consenso quanto ao que se espera nesse tipo de análise exploratória: a classificação de diversas entidades em um número reduzido de grupos mutuamente excludentes. Em outras palavras, os agrupamentos resultantes deverão exibir elevada homogeneidade interna e elevada heterogeneidade externa. ${ }^{14,12,15}$ Assim, para facilitar a análise de cluster e visualizar o agrupamento dos setores foi elaborado um dendograma com auxílio do pacote estatístico Stata versão 9.0.

\subsection{Análise Espacial}

Já para verificar se a localização dos lotes de domicílios com indivíduos infectados ocorreu aleatoriamente ou se houve algum padrão de distribuição sistemático pelo território, foi adotada uma mescla bastante utilizada de dois procedimentos de análise espacial de eventos, a "visualização-exploratória". ${ }^{16}$ Nessa perspectiva, buscou-se uma integração dos procedimentos de visualização, ou seja, das ferramentas usadas para mapear dados espacialmente georreferenciados, com os procedimentos exploratórios, que analisam os dados na tentativa de detectar padrões, identificar aglomerados, etc. ${ }^{16}$

Outra análise realizada foi a da densidade de pontos que, tem na estimativa Kernel a técnica mais conhecida e mais utilizada para estimar densidade de eventos. Além de constituir uma boa alternativa para geração de superfícies suavizadas e ser particularmente útil na aproximação de dados socioeconômicos, ${ }^{17}$ o estimador Kernel desempenha um papel importante no contexto epidemiológico por identificar a concentração de casos. ${ }^{18}$ Para tanto foi utilizado o Sistema de Informações Geográficas (SIG) Terra View, que possibilitou a análise espacial de dados de área e de dados de padrões pontuais, num trabalho de operações entre camadas.

Por fim, os dados socioeconômicos que levaram ao agrupamento dos setores censitários foram exportados para o banco de dados geocodificados e incrementados com os resultados da análise espacial, ou seja, sobrepostos aos mapas de Kernel com as densidades de casos confirmados de dengue pelo território. 


\subsection{Aspectos Éticos}

Este trabalho foi aprovado pelo Comitê de Ética em Pesquisa da Universidade Federal de Alfenas, conforme protocolo $n^{\circ} 158 / 2010$. O mesmo não teve apoio financeiro, nem material e não apresenta conflito de interesses.

\section{Resultados}

De acordo com o Instituto Brasileiro de Geografia e Estatística - IBGE, o município de AlfenasMG conta com 70 setores censitários urbanos, pelos quais se distribuem residências de todas as classes sociais. Na Análise de Cluster optou-se, empiricamente, pelo agrupamento das unidades censitárias em quatro grupos. Na Figura 1 é apresentado o dendograma, onde pode ser visualizado o ponto de corte assumido para agrupar os setores, conforme os parâmetros socioeconômicos.

Os setores de número 04 e 70 foram excluídos de forma automática no momento do agrupamento hierárquico e aparecem nos resultados como "sem informação" socioeconômica. Seus parâmetros não foram calculados devido à falta de informação de algumas variáveis do Censo. O setor 04 é um setor especial que foi considerado pelo IBGE como "Setor especial de asilos, orfanatos, conventos, hospitais, etc. sendo constituído de no mínimo 50 moradores". Essa classificação confere com o convento e o ambulatório de especialidades existentes nessa área. Já no setor 70, existe um Residencial à beira do Lago de Furnas, chamado Porto Seguro, em que não há dados da variável "V1138 - Mulheres responsáveis por domicílios particulares permanentes". A ausência dessa informação interferiu anulando os parâmetros "Média dos anos de instrução das mulheres responsáveis pelos domicílios"; "Renda média (em reais*) das mulheres responsáveis pelos domicílios"; "Proporção de mulheres responsáveis analfabetas (\%)". Além disso, outros parâmetros que apresentaram valor zero foram: "Proporção de domicílios com 5 ou mais moradores (\%)"; "Proporção de domicílios com água encanada (\%)"; "Proporção de domicílios com esgotamento sanitário (\%)" e "Proporção de domicílios com coleta regular de lixo $(\%)$.

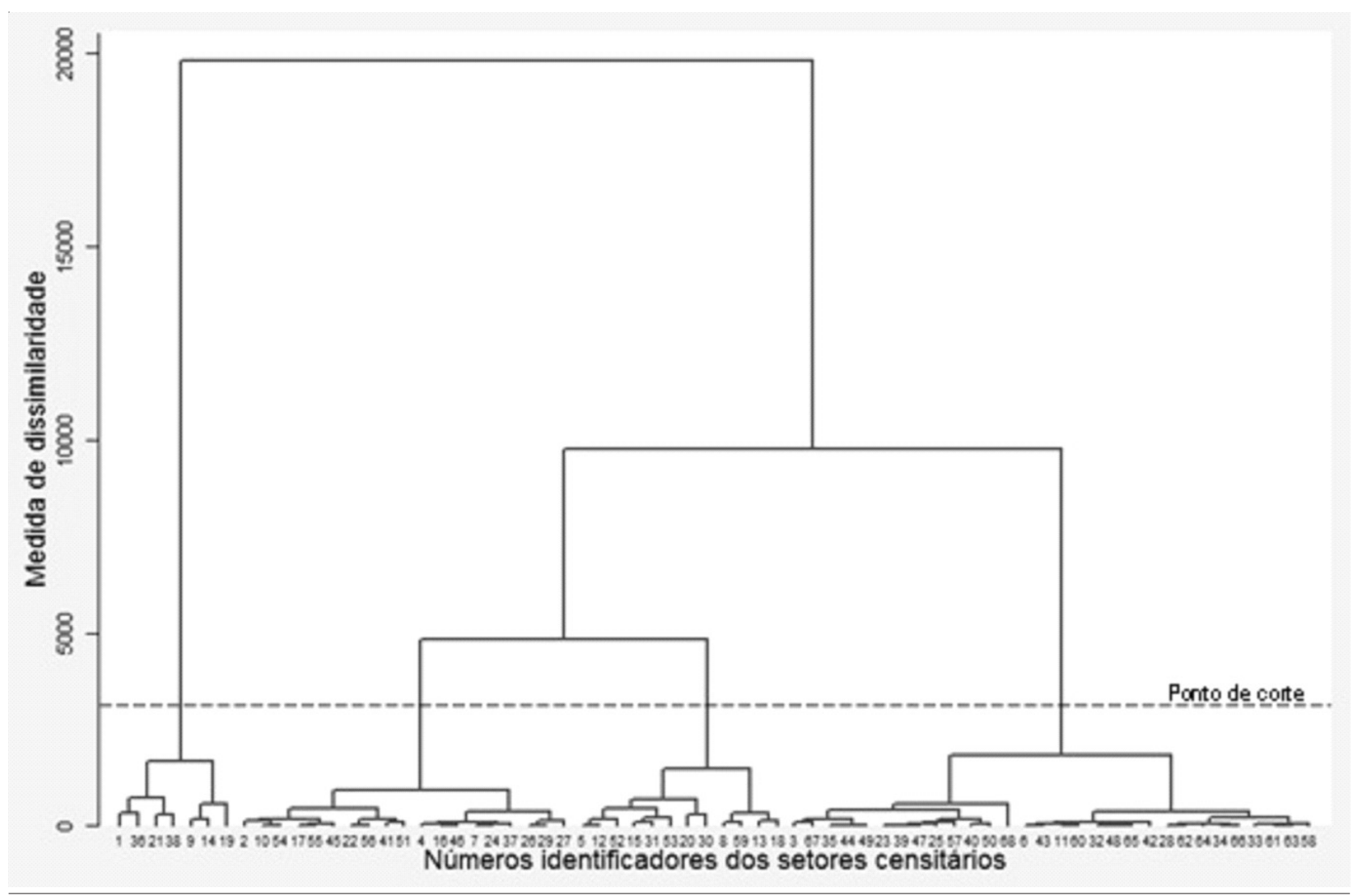

Figura 1: Dendograma para Análise de Cluster, elaborado pelo método do Agrupamento Hierárquico de Ward. 
Ao término desse agrupamento, os valores médios dos parâmetros socioeconômicos dos setores que compunham cada cluster foram calculados, com a finalidade de classificá-los quanto ao nível socioeconômico. Dessa forma, o cluster que apresentou valores maiores foi considerado como de melhor nível socioeconômico, o grupo com valores menores foi classificado como pior extrato socioeconômico e os demais agrupamentos configuraram os níveis socioeconômicos intermediários. A partir desse critério, as unidades censitárias do cluster 1 passaram a compor o nível socioeconômico 1; os setores do grupo 4 figuraram o nível socioeconômico 4 e os setores dos grupos 2 e 3 foram denominados nível socioeconômico 3 e nível socioeconômico 2 , respectivamente (Tabela 1).

Em relação aos casos de dengue, ocorreram 489 notificações durante a década estudada, sendo que 476 remetiam a pessoas com moradia urbana $(97,34 \%)$ e 13 registros indicavam sujeitos residentes na área rural $(2,66 \%)$. Nas notificações de casos urbanos, percebeu-se que $37,18 \%$ resultaram na confirmação de dengue clássico, o que correspondeu a 177 indivídu- os. Foi possível geocodificar $80,22 \%$ dos endereços de casos confirmados por meio de ligação automática, o que equivaleu a 142 fichas de casos de dengue clássico.

A análise espacial mostrou que os locais de residência dos casos formaram diversos aglomerados com distribuição heterogênea pelo território urbano, ano a ano. Ao sobrepor o mapa de pontos e densidade da dengue com o mapa temático dos setores censitários, segundo a classificação socioeconômica, pôde-se perceber que casos confirmados de doença se distribuíram de forma independente ao extrato a que pertenciam. Ou seja, a dengue esteve presente tanto nas regiões com melhor perfil socioeconômico quanto nas áreas menos favorecidas quanto ao saneamento ambiental, às condições de renda, à escolaridade e ao adensamento populacional.

Tal variação espacial, onde os aglomerados ora se situaram em setores com melhor condição socioeconômica (áreas com representação coroplética mais clara), ora em regiões menos favorecidas (áreas com representação coroplética mais escura), pode ser observada no mapa da Figura 2.

Tabela 1: Valores médios dos parâmetros socioeconômicos utilizados para classificar os setores censitários e clusters quanto ao nível socioeconômico.

\begin{tabular}{|c|c|c|c|c|}
\hline \multirow[b]{2}{*}{ Parâmetros Socioeconômicos } & \multicolumn{4}{|c|}{ Média dos parâmetros socioeconômicos } \\
\hline & $\begin{array}{c}\text { Cluster } 1 \\
\text { Nível socio- } \\
\text { econômico } 1\end{array}$ & $\begin{array}{c}\text { Cluster } 2 \\
\text { Nível socio- } \\
\text { econômico } 3\end{array}$ & $\begin{array}{c}\text { Cluster } 3 \\
\text { Nível socio- } \\
\text { econômico } 2\end{array}$ & $\begin{array}{c}\text { Cluster } 4 \\
\text { Nível socio- } \\
\text { econômico } 4\end{array}$ \\
\hline $\begin{array}{l}\text { Média dos anos de instrução das } \\
\text { mulheres responsáveis pelos domicílios }\end{array}$ & 10,1084 & 6,2823 & 7,5964 & 3,8330 \\
\hline $\begin{array}{l}\text { Renda média (em reais*) das pessoas } \\
\text { responsáveis pelos domicílios }\end{array}$ & 2392,6266 & 748,9242 & 1158,2868 & 452,5712 \\
\hline $\begin{array}{l}\text { Renda média (em reais*) das mulheres } \\
\text { responsáveis pelos domicílios }\end{array}$ & 1548,3713 & 460,9510 & 810,8538 & 262,9662 \\
\hline $\begin{array}{l}\text { Proporção de pessoas responsáveis } \\
\text { analfabetas (\%) }\end{array}$ & 0,0145 & 0,0751 & 0,0394 & 0,1525 \\
\hline $\begin{array}{l}\text { Proporção de mulheres responsáveis } \\
\text { analfabetas (\%) }\end{array}$ & 0,0186 & 0,1306 & 0,0693 & 0,2383 \\
\hline $\begin{array}{l}\text { Proporção de domicílios com } 5 \text { ou mais } \\
\text { moradores (\%) }\end{array}$ & 0,2170 & 0,2441 & 0,2186 & 0,2960 \\
\hline $\begin{array}{l}\text { Proporção de domicílios com } \\
\text { água encanada (\%) }\end{array}$ & 0,9970 & 0,9959 & 0,9981 & 0,9524 \\
\hline $\begin{array}{l}\text { Proporção de domicílios com } \\
\text { esgotamento sanitário (\%) }\end{array}$ & 0,9953 & 0,9914 & 0,9970 & 0,9322 \\
\hline $\begin{array}{l}\text { Proporção de domicílios com } \\
\text { coleta regular de lixo (\%) }\end{array}$ & 0,9986 & 0,9943 & 0,9973 & 0,9466 \\
\hline
\end{tabular}




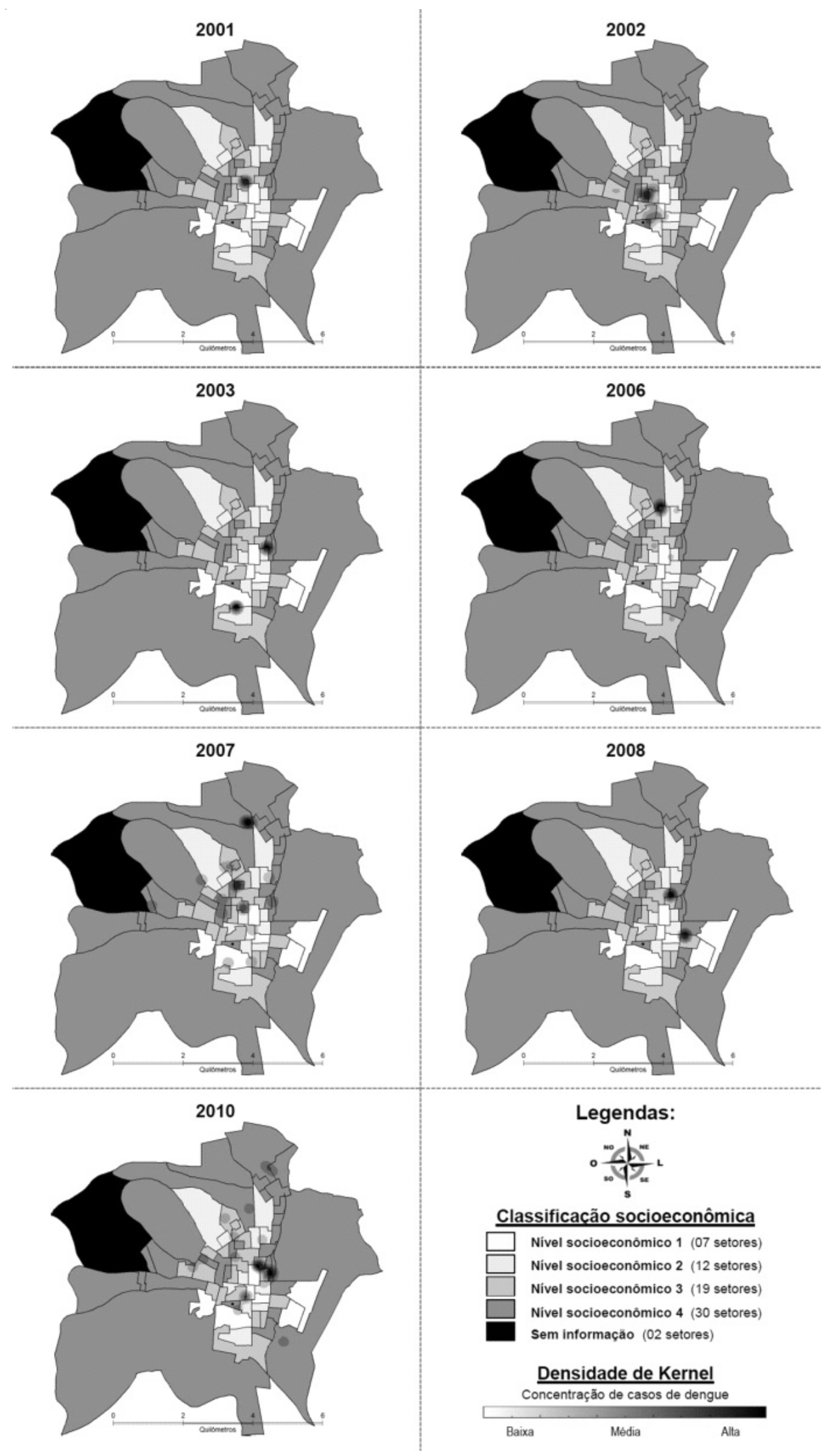

Figura 2: Concentração de casos confirmados de dengue clássico entre 2001 e 2010 (excluídos os anos de 2004, 2005 e 2009) pelos setores censitários agrupados por níveis socioeconômicos. 
As imagens do cruzamento entre as estimativas de Kernel e a malha de setores, segundo o nível socioeconômico, dos anos de 2004, 2005 e 2009, não foram expostas, uma vez que nesses períodos não houve registro de casos confirmados de dengue.

Em 2002, ano epidêmico que apresentou a maior densidade de casos da série, os setores da região central do município foram os mais atingidos. $\mathrm{O}$ setor 8 foi o que apresentou maior concentração (nível socioeconômico 3), seguido pelo setor 9 (nível socioeconômico 2). Já com menor intensidade, foram atingidos outros setores do nível socioeconômico 3, como o 27, o 46 e o 2; áreas de nível socioeconômico 2, como os setores 6 e 19; de nível socioeconômico 4, como os setores de número 3 e 7 ; e de forma mais discreta em setores de melhor nível socioeconômico, como o 1,15 e 10.

A analisando todo o período, o nível socioeconômico 3 foi o que apresentou o maior números de casos, seguido pelos extratos 4, 2 e 1. Quando o foco da observação foi a proporção de domicílios com casos de dengue, pôde-se perceber que as classes socioeconômicas 1 e 3 foram as mais acometidas, conforme exposto na Tabela 2.

Já atentando para a caracterização dos setores de forma individualizada e levando em conta todos os casos de dengue de 2001 a 2010, percebeu-se que os setores censitários que contiveram o maior número de casos foram os de número 08, 02 e 27 . Em contrapartida, os setores com maior proporção de domicílios com casos de dengue foram os de número 08, 27 e 09 . Interessante notar que, contrariando o pressuposto que a dengue é preponderante em áreas com alta densidade populacional, os setores que apresentaram maior número de casos e proporção de domicí- lios não corresponderam às regiões com maior número de residências.

\section{Discussão}

Para discorrer sobre a relação entre a concentração de casos de dengue e o contexto socioeconômico, o primeiro ponto de discussão é se os quatro agrupamentos de setores censitários realmente representam regiões socioeconomicamente distintas. Sobre esse aspecto, a própria análise dos valores médios apresentados na Tabela 1, referentes aos parâmetros socioeconômicos adotados, auxilia nessa distinção e aponta para diferenças ambientais, de renda, escolaridade e adensamento populacional.

De todos os parâmetros analisados, os únicos que apresentaram valores semelhantemente elevados entre os extratos, foram os de natureza sanitária. Além disso, por meio da visualização espacial, pôde-se notar que a divisão censitária em Alfenas se aproxima dos limites dos bairros. Essas características, somadas a observações de campo, permitem constatar que as diferenças historicamente conhecidas quanto ao poder aquisitivo da população, ao padrão de construção das residências e à paisagem geográfica dos bairros, foram coerentes com os extratos socioeconômicos obtidos.

Assumindo, dessa forma, que a classificação socioeconômica realizada se aproxima da realidade, outra questão a ser analisada é a falta de um padrão de distribuição sistemática de casos de dengue pelo território. Se por um lado, a visualização espacial das concentrações de casos de dengue, de cada ano separadamente, apontou uma localização independe do extrato socioeconômico, ao se considerar o conjunto

Tabela 2: Caracterização dos extratos socioeconômicos quanto à distribuição da dengue pelos domicílios/ setores.

\begin{tabular}{lcccc}
\hline Classificação socioeconômica & $\begin{array}{c}\text { Número de } \\
\text { setores }\end{array}$ & $\begin{array}{c}\text { Número de } \\
\text { domicílios }\end{array}$ & $\begin{array}{c}\text { Casos de dengue } \\
\text { geocodificados }\end{array}$ & $\begin{array}{c}\text { Proporção de domicílios } \\
\text { com casos confirmados }\end{array}$ \\
\hline Nível socioeconômico 1 & 7 & 1498 & 18 & 0,0120 \\
Nível socioeconômico 2 & 12 & 2807 & 30 & 0,0107 \\
Nível socioeconômico 3 & 19 & 4788 & 56 & 38 \\
Nível socioeconômico 4 & 30 & 7949 & 00 & 0,0048 \\
Sem Informação & 02 & 05 & $\mathbf{1 4 2}$ & 0,0000 \\
\hline Total & $\mathbf{7 0}$ & $\mathbf{1 7 0 4 7}$ & $\mathbf{0 , 0 3 9 2}$ \\
\hline
\end{tabular}


de dados, a impressão já não foi a mesma. O grupo de nível socioeconômico 3 foi o que apresentou o maior número de casos e, no grupo de melhor nível socioeconômico (1), foi percebida maior proporção de domicílios com casos de dengue.

Entretanto, essa análise frequentista por extrato socioeconômico, que atribui uma maior proporção de domicílios com casos dengue ao grupo 1 e sugere uma relação maior entre a ocorrência de dengue e o melhor nível socioeconômico, por exemplo, pode estar enviesada. Para perceber tal distorção, basta verificar que esse nível foi o que apresentou o menor número de casos e a menor proporção de domicílios com pessoas infectadas. Assim, acredita-se que a melhor maneira de analisar o comportamento da dengue pelos grupos, seja separadamente, ano a ano e, nesse sentido, os aglomerados de casos não apresentaram um padrão de distribuição constante em nenhum dos 4 níveis socioeconômicos.

A ausência de associação entre a dengue e o contexto socioeconômico na quase totalidade do período estudado, também foi percebida em outros trabalhos, o que merece ser mais bem investigado, considerando, inclusive, a possibilidade de variação conforme a realidade de cada município. ${ }^{7}$ Acredita-se ainda que a realização de pesquisas sobre condições particulares da doença têm potencial para corroborar o entendimento do papel dos grupos sociais na dinâmica de transmissão da dengue. ${ }^{18}$

Este estudo também foi ao encontro da necessidade de trabalhos que abordem as localizações espaciais de eventos relevantes para a saúde pública e a importância do uso dos Sistemas de Informações Geográficas para a identificação de heterogeneidade espacial em nível local. ${ }^{18}$ Assim, espera-se que este retrato parcial dos aspectos epidemiológicos, geográficos e sociais da dengue em Alfenas-MG possa contribuir para a construção do conhecimento e do enfrentamento da doença na escala municipal.

\section{Conclusão}

O trabalho alcançou seus objetivos, uma vez que o estudo de variáveis referentes ao saneamento ambiental, às condições de renda, à escolaridade e ao adensamento populacional dos setores censitários de Alfenas-MG, possibilitou a classificação do Município em níveis socioeconômicos. Além disso, os dados do processo de visualização-exploratória e da análise da densidade de pontos foram incorporados à estratificação socioeconômica construída, num trabalho de operação entre camadas que resultou na descrição espacial dos casos confirmados de dengue clássico, entre 2001 e 2010, por áreas urbanas heterogêneas do ponto de vista socioeconômico.

Tomados em conjunto, os resultados apontam que não houve relação alguma entre o padrão de distribuição geográfica da doença com os diferentes clusters socioeconômicos do Município. Embora não tenha sido objetivo deste trabalho, investigar aspectos subjetivos da relação "dengue e contexto socioeconômico" acredita-se que tal conhecimento seja igualmente importante do ponto de vista epidemiológico e social. Portanto, sugere-se que sejam desenvolvidas pesquisas que agreguem abordagens qualitativas sobre o problema da dengue no nível local, a fim de corroborar o recorte geoepidemiológico ora apresentado.

\section{Referências}

1.. BRASIL. MINISTÉRIO DA SAÚDE. Programa Nacional de Controle da Dengue: amparo legal à execução das ações de campo - imóveis fechados, abandonados ou com acesso não permitido pelo morador. Ministério da Saúde. Brasília; 2006.

2. Costa MFL, Barreto S, Giatti L. A situação socioeconômica afeta igualmente a saúde de idosos e adultos mais jovens no Brasil? Um estudo utilizando dados da Pesquisa Nacional por Amostras de Domicílios - PNAD/98. Ciênc Saúde Coletiva. 2002; 7: 813-24.

3. Nunes A, Santos JRS, Barata RB, Vianna SM. Medindo as desigualdades em saúde no Brasil: uma proposta de monitoramento. Organização Pan-Americana de Saúde. Brasília; 2001.

4. Duarte EC, Schneider MC, Paes-Sousa R, Ramalho WM, Sardinha LMV, Silva Júnior JB, Castillo-Salgado C. Epidemiologia das desigualdades em saúde no Brasil: um estudo exploratório. Organização Pan-Americana da Saúde. Brasília; 2002.

5. Kawamoto EE, Santos MCH, Mattos TM. Enfermagem Comunitária. São Paulo: EPU; 2004.

6. Tauil PL. Saúde em Foco: informe epidemiológico em saúde coletiva 2002; 24: 47-53.

6. Tauil PL. Dengue: as prováveis causas de sua re-emergência e disseminação. Saúde em Foco: informe epidemiológico em saúde coletiva 2002; 24: 47-53.

7. Mondini A, Chiaravalloti Neto F. Variáveis socioeconômicas e a transmissão de dengue. Rev Saúde Pública. 2007; 41: 923-30.

8. Flauzino RF, Souza-Santos R, Oliveira RM. Dengue, geoprocessamento e indicadores socioeconômicos e ambientais: um estudo de revisão. Rev Panam Salud Pública. 2009; 25:456-61.

9. Departamento de Informática do Sistema Único de Saúde. Informações de saúde [homepage na internet]. Brasília: Ministério da Saúde; 2009. Disponível em: http:// tabnet.datasus.gov.br/cgi/tabcgi.exe?ibge/cnv/poptmg.def [acesso em 10 nov. 2009]. 
10. Instituto Brasileiro de Geografia e Estatística [homepage na internet]. Censo 2000. Brasília: Ministério do Planejamento, Orçamento e Gestão; 2000. Disponível em: http:// www.ibge.gov.br/censo [acesso em 7 maio 2011].

11. Vendramini SH, Santos ML, Gazetta CE, Chiaravalloti-Neto F, Ruffino-Netto A, Villa TC. Tuberculosis risks and socioeconomic level: a case study of a city in the Brazilian southeast, 1998-2004. Int J Tuberc Lung Dis. 2006; 10: 1231-5.

12. Mingoti SA. Análise de dados através de métodos de estatística multivariada: uma abordagem aplicada. Belo Horizonte: Ed. da UFMG; 2005.

13. Pereira JCR. Análise de dados qualitativos: estratégias metodológicas para as ciências da saúde, humanas e sociais. São Paulo: EDUSP; 2001.

14. Hair Junior JF, Anderson RE, Tatham RL, Black WC. Análise multivariada de dados. $5^{\underline{a}}$ ed. Porto Alegre (RS): Bookman; 2005.

15. Ferreira DF. Estatística multivariada. Lavras: UFLA; 2008.

16. Barcellos C, Silva AS, Andrade ALSS. Análise de dados em forma de pontos. In: Santos SM, Souza WV (Org.). Introdução à estatística espacial para a saúde pública. Brasília: Ministério da Saúde, Fundação Oswaldo Cruz; 2007. 3: 29-59.
17. Camargo ECG, Fucks SD, Câmara G. Análise Espacial de Superfícies. São José dos Campos: Divisão de Processamento de Imagens do Instituto Nacional de Pesquisas Espaciais, INPE; 2002.

18. Oliveira EXG. et al. Análise de dados espaciais. In: Santos $S$, Souza-Santos R. (Org.). Sistemas de informações geográficas e análise espacial na saúde pública. Brasília: Ministério da Saúde, Fundação Oswaldo Cruz. 2007; 2: 63-80.

18. Oliveira EXG, Silveira Jr JC, Souza-Santos R, Pina MF, Portugal JL. Análise de dados espaciais. In: Santos S, SouzaSantos R (Org.). Sistemas de informações geográficas e análise espacial na saúde pública. Brasília: Ministério da Saúde, Fundação Oswaldo Cruz. 2007; 2: 63-80.

19. Flauzino RF, Souza-Santos R, Oliveira RM. Indicadores Socioambientais para Vigilância da dengue em nível local. Saúde Soc. 2011; 20: 225-40. 